\title{
Development of Novel Actuating Mechanisms for Smart Artificial Flowers
}

\author{
Chi Hsiang Pan, Chia Hao Lin, Hsin-Ying Pan \\ Department of Mechanical Engineering, National Chin-Yi University of Technology \\ Taichung, Taiwan, ROC \\ pancs@ @ ncut.edu.tw; insistenceon@gmail.com
}

\begin{abstract}
We present novel actuating mechanisms for smart artificial flowers. Artificial flowers are static decorations, but if we make them movable, this will make them more lively and entertaining. The statement "smart" represents the flower that is able to interact with the surrounding and to show dynamic visual effects such as the blooming of flower, the swaying of stem just like light tracking of the sunflower. The flower can be executed with the pre-set program or be remote control by using smart phone or computer. The smart flower is consisted of actuating mechanisms, Arduino controllers, and sensors. In this paper, we focused on the movement of the flower. Instead of using conventional actuating mechanisms to actuate the flower, we use shape memory alloy (SMA) as actuators to avoid complication, large volume and noise generation of the components. Two types of the actuating mechanisms are designed, one is for onedirection bending, and the other is for multi-directions bending. The performances of the novel actuating mechanisms are evaluated through the experiment. The prototype of the smart flowers have been developed and can be realized in various application such as robotic flowers, flower lamps, fashion clothing accessories, and decorations in home or offices.
\end{abstract}

Keywords: Smart Artificial Flower, Shape Memory Alloy, Actuating Mechanism.

\section{Introduction}

A smart artificial flower is an intelligent electromechanical device with the appearance of a common flower, with components such as petals, stems and leaves. The smart flower is capable of movements using its actuators to mimic the blooming of flower, the swaying of the stem and the stirring of the leaves in the wind. Besides, the smart flower can recognize the environmental conditions such as temperature, humidity, sound and light intensity, which can create a pleasing and interactive phenomenon. A variety of applications of the smart artificial flower are developed, such as the robotic flower (or flower Robot)[1-9], the flower lamp[10], the flower humidifier[11], illuminated flower[12], temperature sensitive flower[13], scent emitting flower[14] and the dynamic artificial plant[15-17]. Give a brief introduction of some examples. The Japanese company TOMY released their product "Flip Flap" [17], a small artificial plant functioned by solar energy, its two round thick leaves go up and down when there is light shining above, it became popular with its delightful and cute looking, so people would put it in their home or offices as a decoration. The French designer Patrick Jouin[10] in 2012 published a lamp with dynamic lampshade like flower blooming which changes the light brightness, not only making appearance beautiful, but also increase creativity in lighting design. Jiang proposed a Bionic Humidifier [11], which the flower will perk back up and resume humidifying when you provide water. This apparatus has been created to live, die and be maintained like a flower, making it a "natural breeze effect in home humidifying." Joanna Berzowaska's Kukkia dress [18-20] put bionic flowers around the neck of the dress which the flower blooms every 15 seconds, resulting in dynamic visual effects. David Holstius[15] proposed a practice by combining living plants with sensors and lights in an interactive display, and by creating a robotic analogue that mimics phototropic behaviour. Another famous application of the smart artificial flower is robotic flower (or flower robot) which look just like a flower and could actually act like a robot one. It has diverse features include moving, sensing, fragrance emitting, etc. Flower robots can be said as biologically-inspired robots that they are suitable for home application, decorating, entertaining and a close friend of human beings. Because of the above high-quality creative devices, they make the facilities and environmental space resulting in an unexpected additional value and artistic conception.

Most of the smart artificial flowers are powered by conventional motors and they are transferred into flower movements through means of gear box, pulley cable or tendon mechanism, etc. For examples, Carnegie Mellon University researchers [1] in 2007 developed a flower Robot, which has moving functions and sensing functions. This flower robot was actuated 
by DC servo motors and has IR sensors on three of its petals so it can track objects moving in front of it. It can also catch a lightweight ball, the flower blooms and dies with the sunshine. Woo-young Kim et al., [2] proposed the mobile flower robot which has flower-type design, mobility, and localization. The flower robot can mimic the swaying of branches and the blooming of flowers, which were actuated by tendon mechanisms and DC motors. Besides, the flower robot has a mobile platform which consists of three-wheels with the steering ability, respectively. Nguyen Truong-Thinh et al [3] in 2011 proposed a flower robot with biomimetic structure based on "tendon and spring" mechanisms. The tendon is made of strong plastic string. The mechanical structure is designed specifically to help the flower has softness and flexible movement. Two DOF motion for robotic stem was realized by the tendon mechanism combining flexible spring actuated by 3 servo motors, and 1-DOF motion of flower was created by a servo motor. It can also sense the movement of the nearby subjects. Huang et al [4] in 2007 used DC micro motors and tendon mechanisms to move whole parts of flower robot such as flower, stem and leaf. Lindsay Sanneman et al [5] proposed a pouch-actuated robotic flower, which has one connector, wires, a tube, a printable and inflatable pouch, and LEDs. The pneumatic and electrical system below the acrylic tile provides air pressure and electric signals to the pouch and LEDs in the flower. Besides the conventional motors as actuator, the shape memory alloy (SMA) is also used as actuating element. Huang et al [6] in 2007 they focused on the swaying of the stem structure, authors adopted coil type SMA as an actuator and then proposed silicone stem structure with 3 coil type SMA. They designed and fabricated the stem structure with $8 \mathrm{~mm}$ of diameter and 50mm of length. After a year, in 2008[7], they proposed silicone stem, petal and leafage structure used 6 coil type SMA, and there were embedded 3 coil type SMA actuators in a silica gel rod. In patent CN2115908U [8] there was an artificial flower, which is automatically open and close according to the change of environment temperature. Petals and leaves of the artificial flower are made of bidirectional shape memory alloy generate reversible deformation for the change of environment temperature, and the bidirectional shape memory alloy is combined on the surfaces of the leaves and the petals. The microcontroller-powered robotic flower "Kasubana" [9] was created that could bloom based on nitinol shape memory alloy actuation and detect human proximity using electric field imaging and capacitance sensing. The flower body houses five actuation joints that connect the five petals to the main stem with five nitinol wires. When current is passed through the nitinol wires, the wires contract and straighten out like a muscle, pushing the petals downward. Thus, the flower opens. A stepper motor was the driving force behind the flower's ability to orient itself in various directions.

\section{Concept Design of the Smart Flower}

The presentation of this research is mainly based on our previous researches [21-24]. The "smart artificial flower" represents the flower that is able to interact with the surrounding such as temperature, humidity, voice and light intensity, and to show dynamic visual effects such as the blooming of flower, the swaying of stem just like light tracking of the sunflower. The flower can be executed with the pre=set program or be remote control by using smart phone or computer. The flower is consisted of floral elements, actuating mechanisms, Arduino control elements, and sensing element, as shown in Fig. 1. Coupled with controllers and different sensors and programs, the smart flower can perform a variety of interactive performances, and even using smart phones and computers for remote control. As for controllers, we use Arduino is because that it is open source and inexpensive, IDE (Integrated Development Environment) is available for free download and is easy to learn to use. Arduino controllers are available in a variety of official or compatible versions. The Arduino controller [25] is based on the open source $\mathrm{I} / \mathrm{O}$ interface. It can be connected to a variety of electronic devices and communication modules, and using Arduino language to write code in the Arduino IDE. As for sensors, ambient light sensors, sound sensors, temperature/humidity sensors and proximity sensors, etc., can be used to achieve intelligent performance of the smart flower. Through these sensors the product can interact with environmental conditions and people. Besides, we can make smart flowers to show performances through Bluetooth or Wi-Fi by using smart phone, iPad or notebook.

In this paper, we focused on the movement of the flower, and the movement is operated by SAM actuating mechanisms and is controlled by Arduino controllers, and various sensors. For example, when someone is approaching, the flower blooms due to the proximity sensors and the control program. For another performance: the flower senses the light coming from the surrounding and then the stem bends just like light-tracking of a real sunflower, and these actions will create a variety of friendly, entertaining and pleasant atmosphere. 


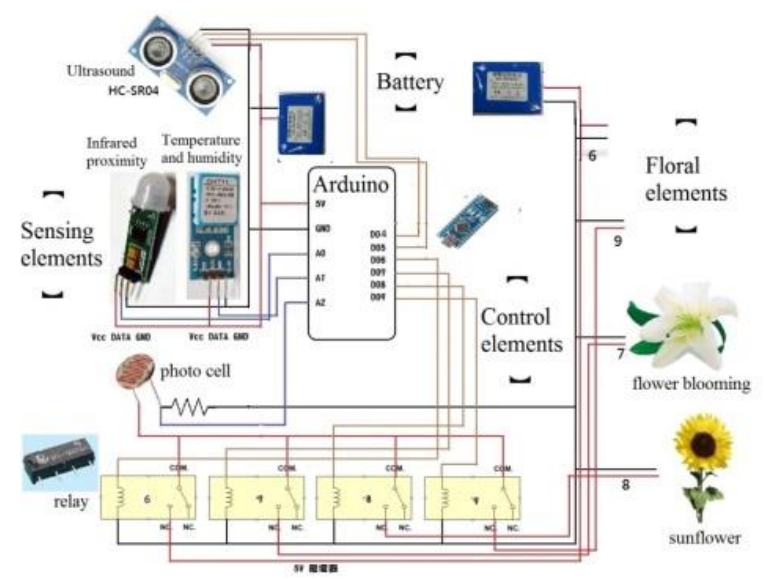

Fig. 1: Construction of the smart artificial flowers.

\section{Shape Memory Alloy Actuating Elements}

Instead of using conventional actuating mechanisms to operate the flower for flower blooming and the stem swaying, we use shape memory alloy (SMA) as actuators to avoid complication, lager volume and noise generation of the components such as motors, pulleys, links and gears. Although response time for a shape memory alloy (SMA) is comparatively slow in cyclic applications, but it is appropriate for the movement of a flower. SMA used as an actuating element has the following intrinsic characteristics [26]: large stroke length/weight ratio and high power/weight ratio (i.e., lightweight and powerful), flexibility in designing motion directions and different shapes, can be driven by thermal or current heating at a specified temperature, quiet operation that is completely free of both electrical and acoustic noise, and can be used where cleanliness is critical. Since SMA actuating elements do not require the use of extra components, SMA actuators can reduce the size of actuators by at least two or three orders of magnitude compared to conventional actuators (for example, hydraulic, pneumatic and electric actuators).

\subsection{Helical Spring-shaped SMA Actuating Element}

The SMA used in this paper is a unidirectional Ti-Ni SMA wire with one way shape memory effect, $0.30 \mathrm{~mm}$ in diameter and the metamorphic temperature is $50^{\circ} \mathrm{C}$. The use of SMA wire for a specific application may require a piece of SMA to be moulded into the required shape. The characteristic heat treatment is then performed to set the specimen to its final shape. Helical spring-shaped SMA (Fig. 2) is fabricated for the blooming of the petals and swaying of the stems. Fabrication process steps for the helical spring-shaped SMA and the performance measurement can be referred to [21-24].

Fig. 2: Helical spring-shaped SMA actuating element.

\subsection{Measurement of the Performances of the SMA Actuating Element}

Through a micro-force measurement set [21-24], the pull force( $\left.\mathrm{F}_{\mathrm{P}}\right)$, the recovery (or restoring) force(Fr) of the SMA actuating elements can be measured. The pull force means the force that is required to elongate the helical-shaped SMA actuating element. The magnitude of the pull force is depended on the elongation length $\left(\mathrm{E}_{\mathrm{L}}\right)$, as shown in Fig. 3. The recovery force $(\mathrm{Fr})$ means the force that is generated when the SMA actuating element contracts into its original length. The magnitude of the recovery force is depended on the contraction length $\left(\mathrm{C}_{\mathrm{L}}\right)$, as shown in Fig. 4. When heated, the SMA actuating element contracts and returns to its original shape, thus acting like a muscle. Because current must flow through the SMA to heat it, a solid metal connection is essential. We crimped the two ends of a SMA wire with electrical wire using metal joints as shown in Fig. 5. A regulated K TYPE thermal couple is provided for temperature measurement of the SMA, and a timekeeper is used to measure response (or recovery) time. 


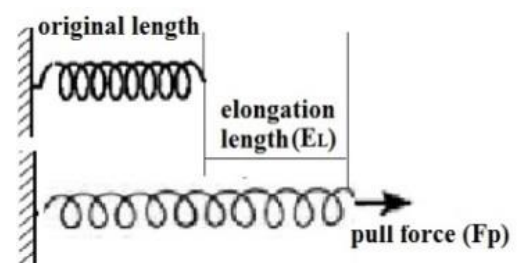

Fig. 3: pull force measurement.

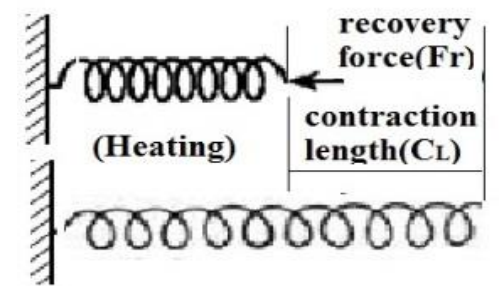

Fig. 4: recovery force measurement.

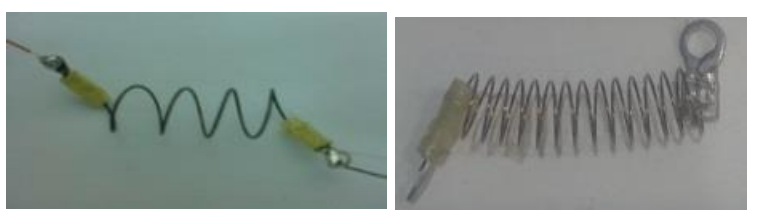

Fig. 5: Connecting helical shaped SMA element and electrical wire via crimps (metal joints).

\subsection{Measurement Results}

From the results, it is obvious that a large drive current will produce large recovery force, high temperature and has faster response (or recovery). But it is found that the time required for heat dissipation of the SMA actuating element to its initial temperature is almost the same in different drive currents through the same SMA element. It is found that when a helical shaped SMA actuating element is pulled, the amount of elongation length is longer, the recovery force is larger, but the recovery speed is the same no matter what elongation length is to be. Figure 6 shows the relationship between the recovery force $(\mathrm{Fr})$ and the pull force $\left(\mathrm{F}_{\mathrm{P}}\right)$ with respective to the elongation length $\left(\mathrm{E}_{\mathrm{L}}\right)$, and Figure 7 shows the relationship between the recovery force $(\mathrm{Fr})$ and the contraction length $\left(\mathrm{C}_{\mathrm{L}}\right)$. It is obvious that it is required larger pull force to produce more elongation length, and it produces larger recovery force when elongation length is large. Besides, the response time and the cooling time are decreased in the same elongation length and with the same drive current as the SMA element become shorter.

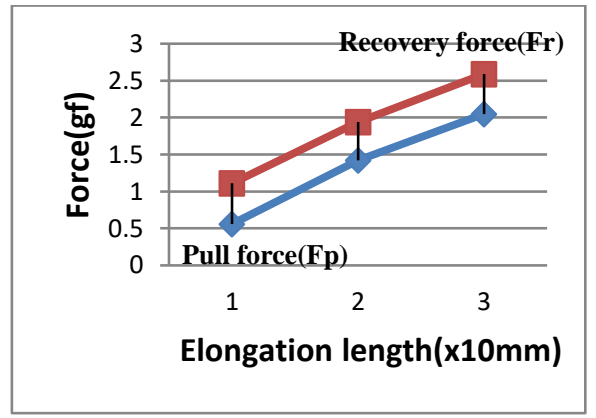

Fig. 6: The pull force $\left(\mathrm{F}_{\mathrm{P}}\right)$ and the recovery force $(\mathrm{Fr})$ with respective to the elongation length $\left(\mathrm{E}_{\mathrm{L}}\right)$. 


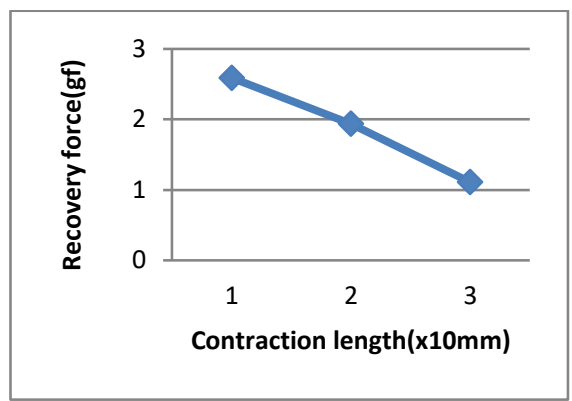

Fig. 7: the relationship between the recovery force $(\mathrm{Fr})$ and the contraction length $\left(\mathrm{C}_{\mathrm{L}}\right)$.

\section{Design and Fabrication of Novel Actuating Mechanisms}

Based on the helical spring-shaped SMA element, we designed different actuating mechanisms for the blooming of the flower and the swaying of the stem. Two types of novel actuating mechanisms are designed and fabricated. One actuating mechanism is designed for one-direction bending. The other is designed for multi-directions bending. The main components of the actuating mechanisms are the helical-shaped SMA actuating element, the metal tube (pipe) spring and the thin plastic plates. The tube spring is $3 \mathrm{~mm}$ outside diameter and the wire diameter is $0.3 \mathrm{~mm}$, elasticity of $33 \mathrm{~N} / \mathrm{m}$ and with good flexibility in bending, as shown in Fig. 8. Figure 9 shows the recovery force generated by the tube spring when it bends and returns to straight shape. The length of the tube spring is $10 \mathrm{~mm}$ for test example. It is measured by the micro-force measurement set. One end of the tube spring is fixed, and the other end is pulled by the micro-force measurement set.

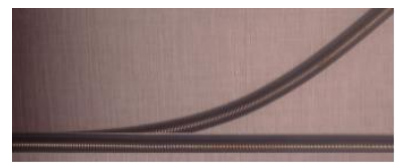

Fig. 8: metal tube (pipe) spring.

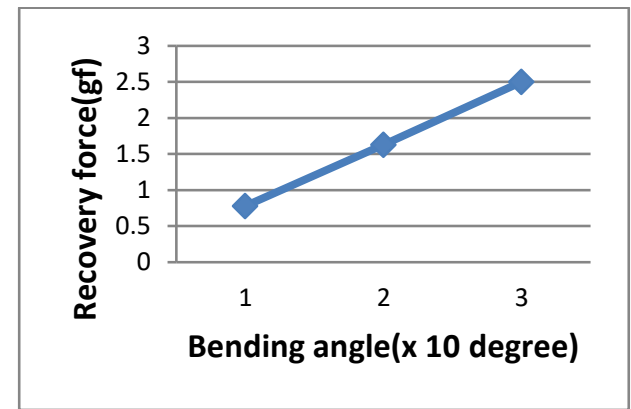

Fig. 9: recovery force generated by the tube spring.

\subsection{The Actuating Mechanism for One-Direction Bending}

Based on the compliant-tube type actuator with 1 D.O.F of bending deflection in previous papers [21, 22], we propose another novel actuating mechanism for one-direction bending, and it is combined with the helical shaped SMA actuating element and the metal tube spring, and both of them are fixed on the two separate thin stiff plates at the front and back sides, as shown in Fig. 10. Ends of the two helical shaped SMA actuating elements are connected together through a metal joint, which the metal joint is fixed on one of the thin plates. The other two ends of the two helical shaped SMA actuating elements are fixed on the other plate respectively. Before the ends of the helical shaped SMA actuating elements are fixed on the two plates, they are pulled into some elongation length. The ends of the tube spring are fixed on two separate plates respectively. The petal can be pasted on the end of the plate. Figure 11 shows the operation of the actuating mechanism for flower petal bending. In operation, the actuating mechanism will deflect due to the shrink (recovery) of the helical shaped SMA actuating elements as heated, and the actuating mechanism will return back to its original straight status depending on the elasticity (recovery force) of the tube spring when heating is stopped. The bending moment is obtained because helical shaped SMA 
actuating elements are offset from the central axis of the thin plates. Figure 12 displays the schematic diagram and the picture of the assembling flower. Figure 13 shows the detailed fabrication processes of the actuating mechanism (prototype) for onedirection bending. The thin plastic plate restricts the actuating mechanism to have only one degree of freedom to deflect. The gap distance of the two separate plates will affect the bending stiffness of the tube spring (i.e. the magnitude of the recovery force generated by the tube spring), and this will affect that how long the elongation length of the helical shaped SMA is. The magnitude of the recovery force generated by the tube spring when it is bending is shown in Fig. 9. According to the pull force and the recovery force of the helical shaped SMA elements (Fig. 6) and the recovery force of the tube spring (Fig. 9), the gap distance of the two plates can be decided. From the experimental results, it indicates that the response time reduces with larger driving current, and it demonstrates that the displacement is in direct proportion to the driving current. For an example, an actuating mechanism with about $12 \mathrm{~mm}$ in width and approximately $60 \mathrm{~mm}$ in length, this actuating mechanism can produce approximately 30 degree bending in $4 \mathrm{sec}$ using $1 \mathrm{~A}$ current and can return to original shape in $7 \mathrm{sec}$.

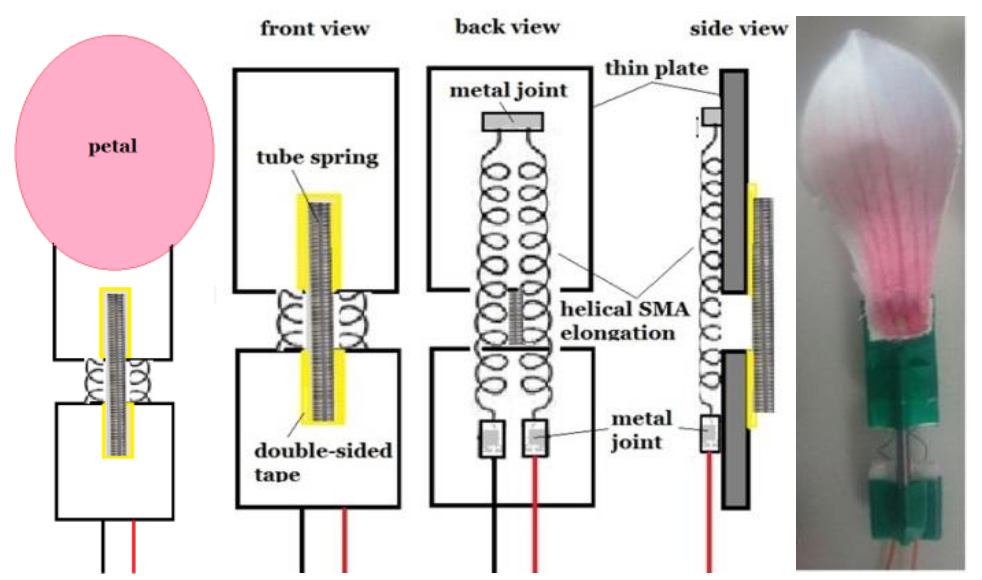

Fig. 10: schematic diagram and picture of an actuating mechanism for one-direction bending.

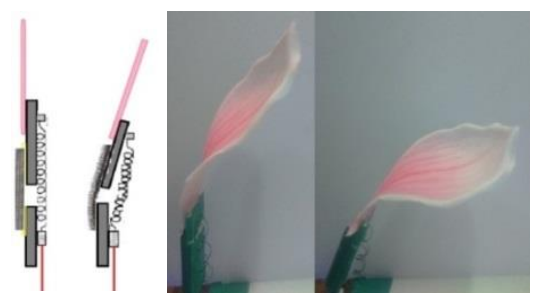

Fig. 11: the operation of the actuating mechanism for flower petal bending.

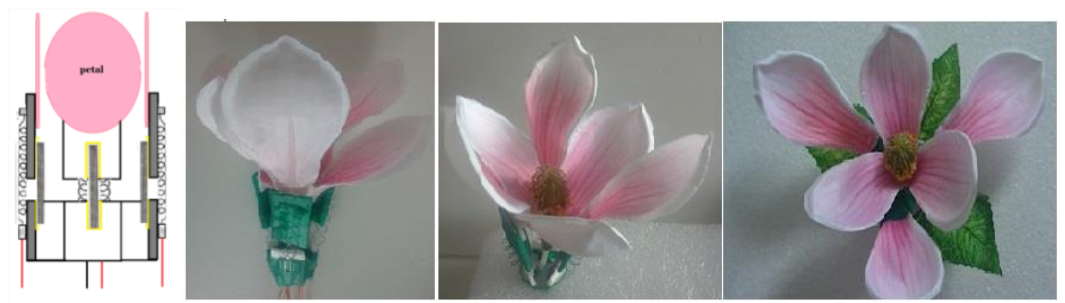

Fig. 12: the shematic diagram and the pictures of the assembling flower. 


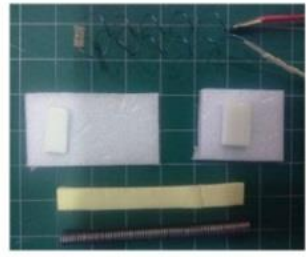

(a)
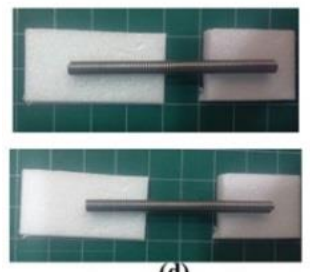

(d)

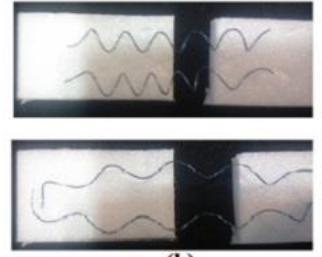

(b)
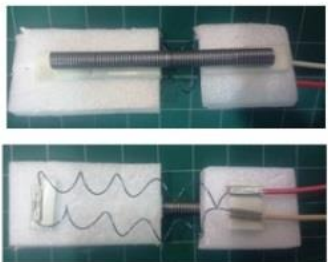

(e)

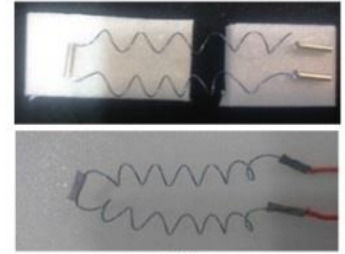

(c)
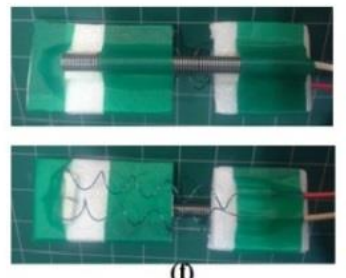

Fig. 13: fabrication processes of the actuating mechanism (prototype) for one-direction bending.

\subsection{The Actuating Mechanism for Multi-Direction Bending}

Based on the actuating mechanism for one-direction bending and the tube-type actuator with 3 D.O.F of deflections in previous papers [21-24], an new actuating mechanism for multi-direction bending is proposed, as shown in Fig. 14. The actuating mechanism is constructed of two adjusting blocks (like a short circular cylinder) with a tube spring in the center and three sets of the helical-shaped SMA actuating elements to provide multi-bending deflections. The construction of the set of the helical-shaped SMA actuating element is similar to the actuating mechanism for one direction bending (Fig. 10). In operation, the mechanism will deflect due to the shrink of one or two sets of the helical shaped SMA actuating elements when heated, and will restore by the tube spring when heating is stop. Because the gap distance of the tube spring and the elongation length of the helical shaped SMA actuating elements will deeply affect the bending characteristic of the mechanism, two adjusting blocks are designed to adjust the two parameters. Therefore, the proper deflection can be obtained for the bending of stem experimentally. For an example, an actuating mechanism with about $10 \mathrm{~mm}$ in diameter and approximately $50 \mathrm{~mm}$ in length, and the gap distance of the tube spring is $10 \mathrm{~mm}$, this actuating mechanism can produce approximately 45 degree bending in $3 \mathrm{sec}$ using 1 A current and can return to original shape in $6 \mathrm{sec}$.
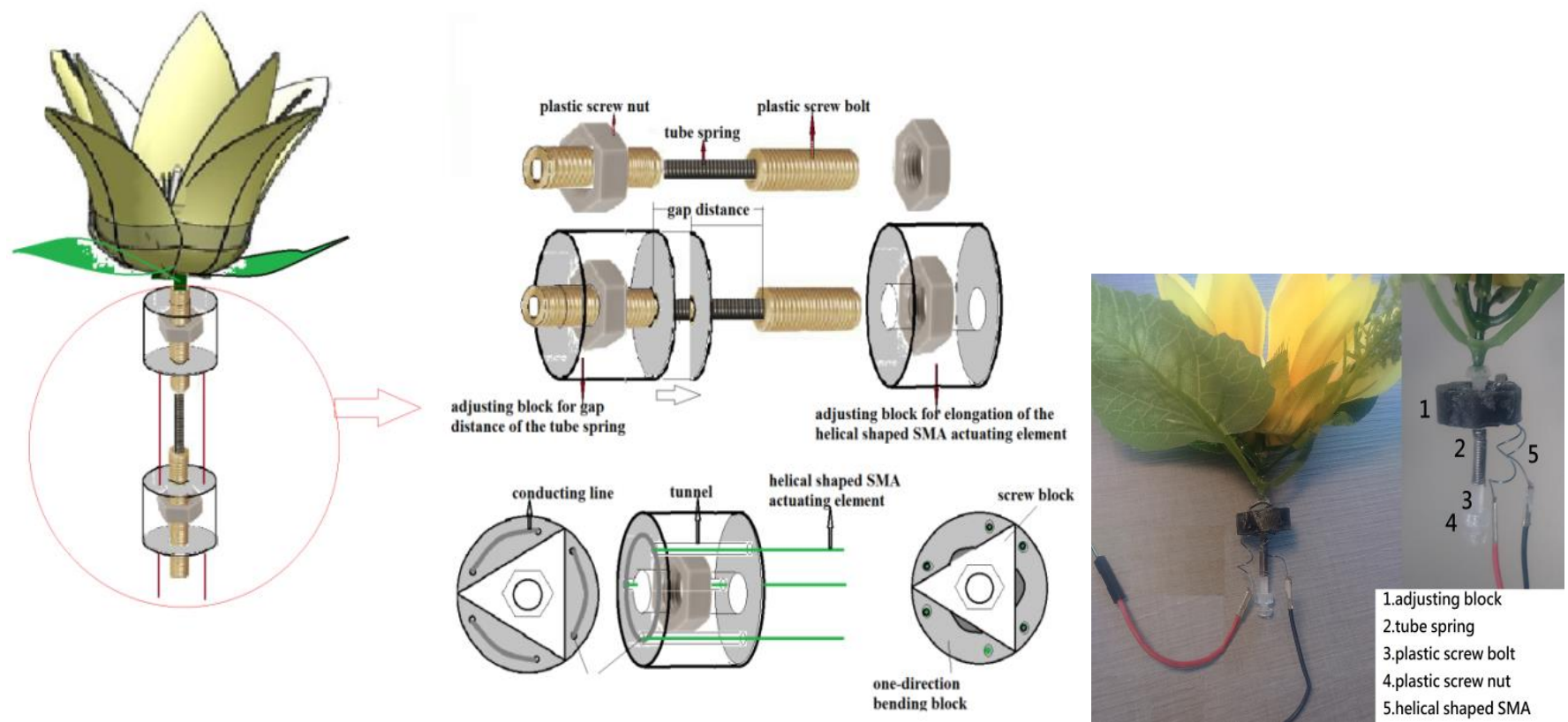

Fig. 14: Schematic diagram and picture of the actuating mechanism for multi-directions bending. 


\section{Conclusion}

We present novel SMA actuating mechanisms for the flower blooming and the stem swaying of the smart artificial flower. The smart flower is operated by SMA actuating mechanism, Arduino controller, and various sensors and is able to sense the signals coming from the surrounding and makes responds, or executed with the pre-set program. Besides, it could be remote control by using smart phone or computer. We use shape memory alloy as actuating elements and pulse width modulation as drive circuit to avoid complication, lager volume and noise generation of the components. The helical shaped SMA actuating elements are made of SMA wire (Ti-Ni) with one way shape memory effect and $0.3 \mathrm{~mm}$ in diameter. Two types of the SMA actuating mechanisms are proposed. One is for flower blooming with one-direction bending, and the other is for stem swaying with multi-directions bending. The prototypes of the mechanisms have been developed, and the performances of the actuating elements and mechanisms have been measured and investigated in experiments.

\section{References}

[1] Plante robot. [Online]. Available: https://fr.wikipedia.org/wiki/Plante_robot

[2] W. Y. Kim, I.H. Song, K. W. Kim, S. H. Park and J. O. Park, "Group Dancing Mobile Flower Robots with Moving Mechanism, Mobility and Localization Functions," Vde Verlag Gmbh, Berlin, Offenbach, pp. 1032-1037, ISBN 9783-8007-3273-9 1033.

[3] T. T. Nguyen and N. P. Nguyen, "Design and Development of Continuum Structure for Robotic flower," Proceedings of the 2011 IEEE International Conference on Robotics and Biomimetics, 2011, Phuket, Thailand.

[4] H. K. Park, S. M. Jung, J. H. Choi, S. H. Park, C. H. Yoon, J. O. Park, "A Study on the Moving Mechanism for Flower Robot," International Conference on Control, Automation and Systems, COEX, Seoul, Korea, 2007.

[5] L. Sanneman, D. Ajilo, J. DelPreto, A. Mehta, S. Miyashita, N. A. Poorheravi, C. Ramirez, S. Yim, S. Kim, and D. Rus, "A Distributed Robot Garden System," IEEE International Conference on Robotics and Automation (ICRA), Washington State Convention Center Seattle, Washington, 2015.

[6] H. L. Huang, S. H. Park, J.O. Park, C. H. Yun, "Development of stem structure for flower robot using SMA actuators," In: IEEE Int. Conf. on Robotics and Biomimetics, ROBIO, 2007.

[7] H. L. Huang, S. H. Park, J. O. Park, "Shape Memory Alloy based Flower Robot," In 39th International Symposium on Robotics, Seoul, Korea, 2008.

[8] CN2115908U, CN Application. [Online]. Available: https://patents.google.com/patent/CN2115908U/en

[9] Kasubana The flower. [Online]. Available: https://people.ece.cornell.edu/land/courses/ece4760/FinalProjects/s2005/yc294_mp282/KASUBANA/index.htm

[10] G. Lidija, (2012), Patrick Jouin's 3D-Printed Bloom Table Lamp Opens Like a Flower. [Online]. Available: http://inhabitat.com/patrick-jouins-3d-printed-bloom-table-lamp-opens-like-a-flower/

[11] Bionic Humidifier, A Flower that Helps You Breathe Easier. [Online]. Available: https://inhabitat.com/bionichumidifier-a-flower-that-helps-you-breathe-easier/

[12] US Patents: US4,399,493.

[13] US Patents: US4,888,219.

[14] US Patents: US6,153,274.

[15] D. Holstius, J. Kembel, A. Hurst, P. H. Wan, J. Forlizzi, "Info tropism: Living and Robotic Plants as Interactive Displays," DIS2004, 2004, Cambridge, Massachusetts, USA.

[16] Breeze the Robotic Tree by Robotany. [Online]. Available: https://www.youtube.com/watch?v=j4d2xvVPlCw

[17] S. Judie, (2004), Tomy Flip Flap Solar Powered Plant Review. [Online]. Available: http://thegadgeteer.com/2004/11/29/tomy_flip_flap_solar_powered_plant_review/

[18] J. Berzowska and M. Coelho, (2005), Kukkia and Vilkas: Kinetic Electronic Garments, Wearable Computers, 2005. Proceedings. Ninth IEEE International Symposium on, pp. 82-85.

[19] J. Berzowska, "Memory Rich Clothing: Second Skins that Communicate Physical Memory," In Proceedings of the 5th Conference on Creativity \& Cognition, ACM Press., pp. 32-40, 2005.

[20] Shape-Memory Applications in Textile Design, Procedia-Social and Behavioral Sciences, vol. 195, pp. 2160 - 2169 , 2015. 
[21] C. H. Pan, Y. S. Jhu, Y. B. Wang, "Development of a Novel Micro-gripper Using Shape Memory Alloy," 5th International conference Mechatronic System and Materials, Vilnius, Lithuania, 2009.

[22] C. H. Pan, Y. B. Wang, "Development of a Novel Micro-actuator Driven by Shape Memory Alloy," Solid State Phenomena, vol. 164, pp. 9-14, 2010.

[23] C. H. Pan, Y. B. Wang, H. Y. Pan, "Development of dynamically artificial flowers driven by Shape Memory Alloy and Pulse Width Modulation," IEEE International Workshop on Advanced Robotics and its Social Impacts, Lyon Convention Center, France, 2015.

[24] C. H. Pan and H. Y. Pan, "Development of Smart and Dynamic Floral Clothing Accessories," 12th International Conference on Mechatronics, Brno, Czech Republic, 2017.

[25] Arduino main web site. [Online]. Available: http://www.arduino.cc

[26] K. Otsuka, C. M. Wayman, Shape memory materials. Cambridge University, 1998. 